Pre-publication final draft of: Steckley, L. (2012). Book review of Therapeutic residential child care for children and young people: An attachment and trauma-informed model for practice by Barton, S., Gonzalez, R. \& Tomlinson, P. British Journal of Social Work, 43(1), 199-201.

\title{
Therapeutic Residential Child Care for Children and Young People: An Attachment and Trauma-Informed Model for Practice
}

Susan Barton, Rudy Gonzalez and Patrick Tomlinson

London, Jessica Kingsley, 2012, pp.287, ISBN 9781849052559 (pb), £22.99

"...I feel a huge responsibility to make sure that every child who is in 'out of home' care has the opportunity to have the choice of experiencing a sense of family and of home - for as long as it takes ... I hope in my lifetime to see the implementation of a more humane system of care of children" (pp.255-6).

And so in 1991, Susan Barton founded the Lighthouse Foundation, an organisation in Victoria, Australia which provides therapeutic residential care to homeless young people aged fifteen to twenty two years old. In this book, Barton, along with her Director Rudy Gonzales and Consultant, Patrick Tomlinson, offer a comprehensive account of the work done in the Lighthouse. From the Therapeutic Family Model of Care, to the details of the practices it comprises, to their robust theoretical underpinnings, all aspects of practice in the organisation are thoroughly discussed. The backgrounds of the young people served there are representative of children and young people in residential child care in many other parts of the world, and the content of this book is relevant to most other residential child care provision.

The authors start with a compelling argument for the importance of theory and its clear connection with practice, and this is particularly useful for combating the antiintellectualism that can sometimes hinder a sound basis for practice in residential child care. The theoretical basis for practice at the Lighthouse Foundation is primarily located in traditions of psychosocial development and the impact of trauma on that development, with an emphasis on attachment, therapeutic containment, and psychodynamic concepts of transference, counter-transference, projection and splitting. Elements of neurobiological and social ecological theories of development, the Sanctuary Model, organisational psychology, systems theory, and even anthropology are also well integrated and usefully applied at relevant points throughout the book. All of the theoretical content is presented coherently, with accessible language and a minimum of jargon.

The chapters that follow then build upon this foundation, deftly weaving relevant theory with tangible practices. A comprehensive account of the impact of trauma is included, as well as a rich chapter addressing the complexities of therapeutic relationships. Case studies, scattered throughout the book, are especially effective in this chapter and offer readers a way into therapeutic practices which may initially seem counter-intuitive (see for example, pp.95-96). The primary focus here is the relationship between carer and young person. It is the provision of compensatory, nurturing relationships, those that can begin to influence young people's internal working models of self and other, that constitutes 'therapeutic'. For this to be possible, those relationships must be robustly supported by all levels of the organisation, and this is thoroughly addressed in the chapter that follows. It is good to see the emotional impact of the work (including vicarious trauma) well addressed, with an especially strong section on staff turn-over and illness that offers useful ways of thinking about and responding to this all too common problem.

The next chapters cover the specifics of the physical and symbolic aspects of the home environment and daily activities of living. The basics of good residential care are covered 
Pre-publication final draft of: Steckley, L. (2012). Book review of Therapeutic residential child care for children and young people: An attachment and trauma-informed model for practice by Barton, S., Gonzalez, R. \& Tomlinson, P. British Journal of Social Work, 43(1), 199-201.

here in detail, though not at all simplistically. Instead, by addressing the theoretical underpinnings and related complexities, the authors reveal how good provision of these basics is anything but simple. The section on routines, limits and anchor points particularly helpful for informing attempts to move away from punitive or controlling approaches while still providing safe, predictable boundaries.

Chapters on community and on group processes offer a strong argument for the importance and practical implementation of a congruent organisational approach to creating the kind of culture that genuinely supports therapeutic care relationships. Key components of a shared language, the impact of trauma re-enactment on the organisation as a whole, and the role of management and leadership in creating the conditions where this can be withstood are well handled. The transition from care into aftercare is covered next, and it was particularly heartening to read in this chapter about the 'life membership' provided to all children who live (or have lived in) Lighthouse Homes. This notion of life membership is predicated on the importance of enduring attachments and the lifetime of involvement many family members share with one another. The book closes with a discussion of outcomes, asking critical questions and addressing related challenges, but ultimately accepting the need to measure and assess the effectiveness of the work. This was one of the few areas where I would have liked to have seen a bit more practical application - in this case, details about which outcomes they measure and how they go about doing it.

From the introduction through the final appendices, I was struck by the constant and integrated presence of thinking, feeling and reflection as integral to meeting the needs of young people, whether at individual or organisational level. While the authors draw from the a therapeutic communities tradition, the content is relevant to the needs of most young people and staff in residential child care, and related practices are applicable to most residential child care settings. Such application may take courage where practice cultures are instrumental in nature, but it is such instrumentality that distorts a clear understanding of the work and ultimately fails children and young people. This book offers vision and motivation to those with requisite courage to work towards a more human system of care for children and young people. 